\title{
EL BANCO DE PROPÁGULOS DE MALEZAS EN EL AGROECOSISTEMA: CONOCIMIENTO ACTUAL Y PROPUESTA METODOLÓGICA PARA SU ESTUDIO ${ }^{1}$
}

\author{
Luis Acosta ${ }^{2}$, Renán Agüero ${ }^{3}$
}

\begin{abstract}
RESUMEN
El banco de propágulos de malezas en el agroecosistema: conocimiento actual y propuesta metodológica para su estudio. El objetivo de este trabajo es revisar los procesos que regulan el banco de semillas de malezas en agroecosistemas, su distribución horizontal y vertical en el suelo, así como proponer una metodología para estudiar el tamaño del banco, potencial de regeneración y lluvia de semillas. En esta revisión se enfoca principalmente los factores internos y externos que regulan la entrada y agotamiento del banco de semillas, el impacto de la labranza sobre los bancos de semillas transitorios y permanentes, y aspectos relacionados con la germinación y latencia de semillas. En los sistemas de producción agrícola el banco de propágulos en el suelo es el recurso primario para las nuevas infestaciones de malezas, el hecho de que una pequeña porción de éste quede remanente en el suelo, asegura su establecimiento cada año. Un conocimiento extenso de los bancos de propágulos y su dinámica, puede proveer la base para el manejo racional de la vegetación espontánea de los agroecosistemas.
\end{abstract}

\begin{abstract}
Propagule banks in agroecosystems: current knowledge and a proposed methodology for their study. The goals with this paper are to review the processes that regulate seed banks in agroecosystems, their horizontal and vertical distribution in the soil, as well as to present a simple methodology for the study of the seed banks size, regeneration potential and seed spreading. We focus mainly on internal and external factors regulating growth and exhaustion of seed banks, the impact of tillage on transitory and permanent banks and aspects related to germination and dormancy of seeds. In agroecosystems, the propagule banks constitute the primary resource ensuring the perpetuation of weeds; even if only a small portion remains, there will be weeds during the next growing season. A broad knowledge of these banks and their dynamics might prove useful for future rational schemes of weed management.
\end{abstract}

\section{INTRODUCCIÓN}

Las plantas para su regeneración requieren que una porción de sus estructuras reproductivas estén en el lugar correcto, en el tiempo adecuado y que sean capaces de germinar dentro de un período limitado en la vida. Así mismo, el tiempo debe coincidir con las condiciones ambientales adecuadas (Cousen y Martiner 1995). El suelo es el medio en el cual estos procesos ocurren en la mayoría de las especies de malezas. Las semillas de malezas constituyen una "memoria" a través de su persistencia en el banco.
Existe variación en la regeneración en las estructuras reproductivas de las distintas malezas, algunas especies germinan a los pocos días de desprendidas de las plantas mientras que otras tienen un período de latencia prolongado (Acosta et al. 1998). La persistencia de las mismas en el suelo depende de diversos factores para la producción y dispersión anual de las semillas (Figura 1). Las semillas que permanecen por períodos prolongados en el banco, presentan una germinación intermitente con longevidades extremas (Murdoch y Ellis 1992). Las malezas son oportunistas en cuanto al uso de estas ventajas para la perpetuación de la especie en

\footnotetext{
1 Recibido para publicación el 24 de mayo del 2001. Proyecto de investigación № 813-A1-802, inscrito en Vicerrectoría de Investigación, Universidad de Costa Rica.

2 Coordinador de Proyectos Prefectura Tarija-Bolivia.

3 Laboratorio de Malezas, Centro de Investigaciones en Protección de Cultivos (CIPROC), Universidad de Costa Rica. E-mail: raguero@cariari.ucr.ac.cr. Tel: (506) 234-6560.
} 


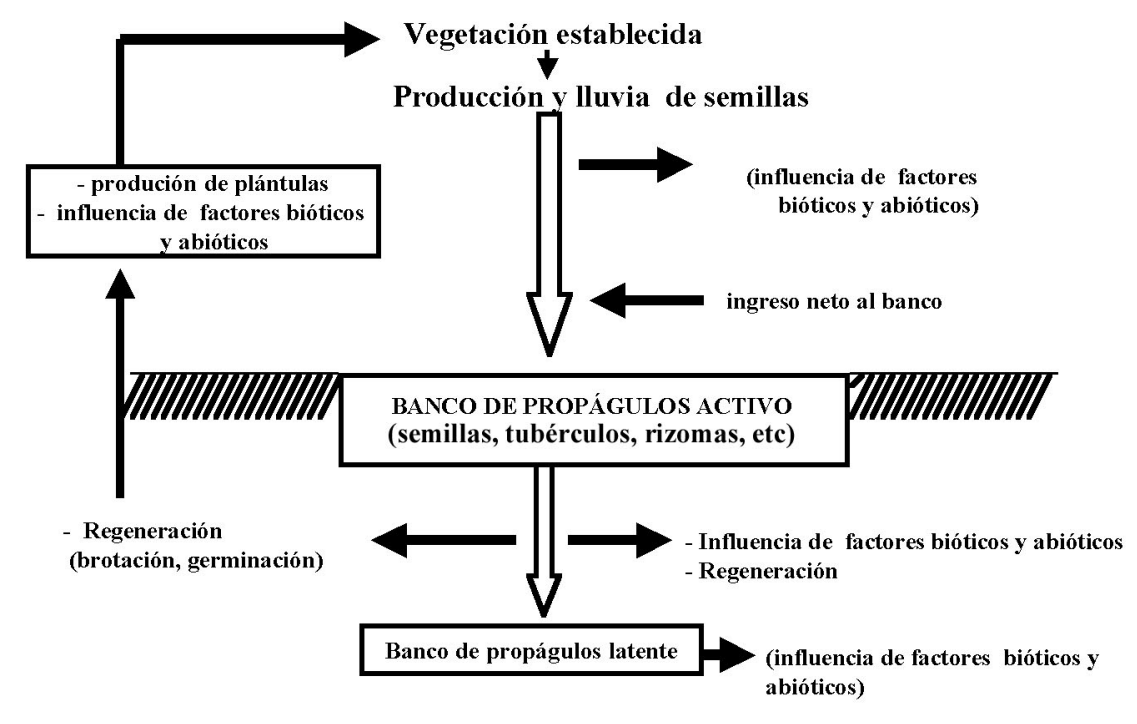

Figura 1. Dinámica de un banco de propágulos.

un lugar determinado. La mayoría de las semillas que germinan durante los primeros años, representan la mayor pérdida de rendimientos en los cultivos, consecuentemente los costos para controlar las malezas también son mayores. En los sistemas de producción agrícola, el banco de propágulos en el suelo es el recurso primario para las nuevas infestaciones de especies de malezas cada año.

Las características del banco de semillas influyen en las poblaciones de malezas que ocurren en el campo y en el éxito de su manejo. Muchos procesos están involucrados en la generación y regulación del banco de semillas de las malezas en el suelo. Las prácticas de manejo tienen un marcado impacto sobre estos procesos, lo cual representa oportunidades para regular las características del banco de propágulos en los agroecosistemas.

El banco de semillas tiene una influencia significativa en las poblaciones de malezas que ocurren cada año en un lugar determinado, lo cual se acentúa en agroecosistemas y por ello, un buen manejo de éste es fundamental para el éxito de un programa de manejo de malezas. El tamaño del banco fluctúa rápidamente dependiendo de la magnitud de la introducción y pérdidas de las semillas de malezas. Muchas de las especies son prolíficas en la producción de semillas, por lo que un control ineficiente garantiza que se recuperen los niveles originales de un año a otro. Recientemente Norris (2000) acuñó el término umbral de cero semillas (UCS), como un concepto de mayor utilidad práctica que el umbral económico. Consiste en evitar la producción de semillas a toda costa.
El propósito de este trabajo es revisar los procesos que regulan el banco de semillas de malezas en agroecosistemas, así como la distribución horizontal y vertical de las semillas en el suelo, para examinar sus efectos y proponer metodologías simples para su estudio.

\section{Entradas de semillas al suelo}

Las semillas entran al suelo de varias maneras, facilitado por la prolificidad de muchas especies. Se ha documentado que una sola planta de Amaranthus retroflexus o de Chenopodium albun cuando crecen sin competencia, puede producir 117400 y 229175 semillas respectivamente, lo que les permite dominar bancos en Colorado y Nebraska (Cuadro 1).

Los ingresos de semillas pueden ocurrir por medio del viento, agua, animales y humanos. Archibold (1980) determinó que semillas de 11 especies de malezas pueden ser acarreadas por el viento e ingresar al banco en número de 2900 por m²; en su estudio Kocia scoparia dominó el banco. Wilson (1988) menciona que el agua de irrigación puede distribuir entre 77 y 137 semillas de malezas de diferentes especies. Así, el agua de irrigación en Nebraska distribuyó hasta 48000 semillas/ha de Amarathus sp. y Chenopodium sp. Kelly y Bruns (1975) realizaron un análisis de aguas del Río Columbia usadas para irrigar pasturas. Se encontraron 22 semillas de Chenopodium y siete de Amaranthus por cada 254 litros. Después de cinco años, estas dos especies se establecieron y llegaron a ser dominantes en el banco de semillas. 
Cuadro 1. Producción y latencia de semillas en el suelo.

\begin{tabular}{lcc}
\hline \multicolumn{1}{c}{ Especies } & $\begin{array}{c}\text { \# semillas } \\
\text { producidas / } \\
\text { planta / ciclo }\end{array}$ & $\begin{array}{c}\text { Sobrevivencia } \\
\text { de semillas } \\
\text { en el suelo } \\
\text { (años) }\end{array}$ \\
\hline Chenopodium album L. & 229175 & 39 \\
Portulaca oleracea L. & 52300 & 40 \\
Ambrosia artemisiifolia L. & 3380 & 40 \\
Rumex crispus L. & 29700 & 80 \\
Taraxacum officinale Weber & 15000 & 6 \\
Setaria viridis (L.) Beauv. & 34000 & 39 \\
Lactuca serriola L. & 27900 & 9 \\
Amaranthus retroflexus L. & 117400 & 10 \\
Helianthus annus L. & 7200 & 1 \\
Bidens frondosa L. & 7000 & 10 \\
Polygonum pensylvanicum L. & 19300 & 30 \\
Capsella bursa pastoris L. & 38500 & 16 \\
Setaria glauca ( L.) Beauv. & 6420 & 30 \\
\hline
\end{tabular}

Fuente: Pollen 2000

Los humanos diseminan semillas de malezas mezcladas con las semillas de los cultivos. Un estudio realizado por Stanelle (1988) encontró que el $29 \%$ de 662 muestras de trigo analizadas contenían semillas de malezas, siendo Avena fatua la especie más frecuente. Por cada kilogramo de semilla de trigo se encontró 1,4 semillas de esta especie, por lo que si se siembra el trigo a una densidad de $67 \mathrm{~kg} / \mathrm{ha}$, se introducirán aproximadamente 94 semillas/ha de esa maleza. Cada planta de A. fatua produce 250 semillas y entonces existirán 23500 semillas para el siguiente ciclo del cultivo. Las semillas de malezas también se dispersan, incluso a largas distancias, cuando la maquinaria sin limpiarla se mueve de un lugar a otro.

Muchas semillas permanecen viables en el tracto digestivo de los animales y pueden dispersarse con las excretas. El tracto digestivo del animal puede proveer un proceso de escarificación. Robert y Douglas (1996) indican que un $14 \%$ de semillas de malezas de cuatro especies permanecieron viables al pasar por el tracto digestivo de tres especies de animales (Cuadro 2). La cantidad de semillas depende de la especie y la calidad dependerá del animal. Panicum mileaceum fue introducido de Nebraska a Colorado, mediante el trasiego de ganado; al cabo de pocos años fue declarada una de las peores malezas en el estado de Colorado (Harmon y Keim 1988).

Los animales silvestres también suelen diseminar semillas de malezas. Se encontró que una especie de paloma (Zenaidura macroura) cazada en un campo in- festado con Panicum mileaceum, consume gran cantidad de semillas de esta Poaceae. Sin embargo, cuando se examinó los intestinos de la paloma para determinar la viabilidad de las semillas, se encontraron pocas semillas que no fueron digeridas (Harvey y Binning 1984). Esas pocas semillas intactas y viables en el tracto digestivo del animal pueden ser suficientes para infestar otros agroecosistemas a lugares distantes. $\mathrm{Al}$ respecto, Parker et al. (1989) opinan que una maleza introducida a un determinado lugar, cuando llega a establecerse y producir semillas, se convertirá en una parte importante del banco de propágulos.

Los factores que influyen la entrada de semillas al banco pueden ser clasificados de la siguiente manera:

1) factores internos:

- producción de semillas dentro del campo (Cuadro 1).

2) factores externos:

- implementos agrícolas

- animales

- viento

- estiércol

- otras actividades humanas

En general, el número de semillas que ingresa al banco por factores externos es muy inferior a aquel producido in situ; sin embargo, las fuentes externas son importantes en el establecimiento inicial de la maleza, en un determinado lugar.

Cuadro 2. Influencia del tracto digestivo de algunos animales domésticos en la viabilidad de las semillas de algunas malezas.

\begin{tabular}{lrccc}
\hline \multirow{2}{*}{ Especie } & \multicolumn{3}{c}{ Viabilidad de las semillas (\%) } \\
& Terneras & Cerdos & Pollos \\
\hline Datura stramonium & L. & 11,3 & 10,3 & 1,2 \\
Convolvulus arvensis L. & 22,3 & 9,0 & 0,0 \\
Ipomoea purpurea L. & 0,3 & 2,3 & 0,0 \\
Solanum nigrum L. & 5,4 & 3,1 & 0,0 \\
\hline
\end{tabular}

Fuente: Robert y Douglas 1996.

\section{Agotamiento de semillas del banco}

El banco de semillas del suelo es fuente de alimento para roedores, insectos y microorganismos; otra porción de semillas germinan y emergen, mientras que otras permanecen latentes o mueren (Brust y House 
1988). La latencia de las semillas es un aspecto medular que garantiza la existencia de bancos; varía considerablemente dependiendo de la especie, profundidad, tipo y preparación del suelo, etc. Kivilaan y Bandurski (1981) mantuvieron enterradas semillas de 20 especies por un período de 50 años. El $42 \%$ de las especies germinaron después de ese tiempo. En otro estudio similar mencionado por Altieri y Liebman (1988), luego de 39 años, 36 especies no germinaron, mientras que otras 25 especies si lo hicieron. Experimentos más recientes sostienen que ciertas semillas en suelos no disturbados tienen un potencial de permanecer viables por períodos de tiempo considerables. Semillas enterradas a $106 \mathrm{~cm}$ permanecen viables por más tiempo que las enterradas a $20 \mathrm{~cm}$. Especies como Setaria sp. y Eriocloa colonum fueron enterradas a $2,5,10$ y $20 \mathrm{~cm}$ de profundidad por un período de tres años; la longevidad de estas especies se incrementó cuando estuvieron enterradas a mayores profundidades (Yenish et al. 1992). Otros estudios realizados por Fenner (1985) y Dyer (1995) demostraron que el disturbio ocasionado al suelo mediante el sistema de labranza redujo un $32 \%$ la viabilidad de las semillas de malezas en comparación con aquellas semillas de suelos no disturbados.

Aunque las semillas tienen un potencial alto de supervivencia en el banco, la mayoría muestran un período de vida relativamente corto en el suelo. En la Figura 1 se indican los eventos de mortalidad y factores que inciden en el agotamiento del banco, tales como:

* germinación

* putrefacción

* predación por animales

* movimiento de semillas

La importancia relativa de estos mecanismos varía con las especies y las condiciones ambientales.

\section{Otras fuentes de salidas de semillas}

Las semillas son fuentes de alimento de:

\author{
* Insectos \\ * Pájaros \\ * Pequeños mamíferos
}

Muchas semillas en el suelo son infectadas por hongos y otros microorganismos. Se han realizado investigaciones para separar los microorganismos que son más eficientes en infectar las semillas del suelo (Crawley 1992, Kremer 1993). Si se llegaran a encontrar patógenos adecuados, éstos podrían ser de gran importancia para inocular los suelos y reducir el tamaño del banco de semillas, siempre y cuando no dañen las semillas de las especies deseables u otros componentes favorables del agroecosistema.

Algunas semillas de malezas pueden ser removidas físicamente del campo por varios mecanismos. El movimiento del agua en el campo es un eficiente distribuidor de las mismas. Campos que son ocasionalmente inundados por ríos o corrientes frecuentemente presentan mayor diversidad de poblaciones de malezas debido a las nuevas deposiciones de malezas. La maquinaria en el campo, particularmente los equipos de cosecha, también puede acarrear semillas fuera y dentro del campo. Pérdidas debido al movimiento generalmente serán pequeñas con relación al total del tamaño del banco de semillas, pero éstas son importantes para empezar nuevas infestaciones de malezas en un determinado lugar.

\section{Dinámica del banco de semillas}

Debido a las entradas y salidas de semillas, el tamaño del banco puede cambiar drásticamente en un período de dos a tres años. Se estima que aproximadamente $2 / 3$ partes del banco de semillas se puede perder cada año por germinación, predación y pudrición. Por lo tanto el potencial de poblaciones de malezas en el campo puede ser reducido con manejo adecuado del banco.

Robert y Douglas (1996) encontraron que en lotes de producción continua de soya, se agotó el $70 \%$ del banco de malezas, luego de tres años con prácticas estándares de manejo (Atrazina + labranza). En cambio, en lotes donde no se usó herbicida durante esos tres años, el banco fue 25 veces mayor. De lo anterior se puede deducir que una pequeña porción de semillas remanente en el banco, es suficiente para nuevas infestaciones, lo cual implica la dificultad para erradicar las malezas ya establecidas en los agroecosistemas. Arauz y Agüero (Figura 2, sin publicar), en un modelo preliminar para Rottboellia cochinchinensis, plantean la hipótesis de que esta especie, a partir de un individuo $/ \mathrm{m}^{2}$, puede reponer el banco de manera exponencial en poco menos de siete ciclos, aún sometida a estrategias de manejo que eliminan arriba del $90 \%$ de las plántulas que emergen durante cada ciclo.

\section{Impacto de la labranza sobre el banco de semillas}

La labranza puede influir significativamente en la densidad y distribución de las semillas dentro del banco; así, cambios en las poblaciones de malezas son fre- 


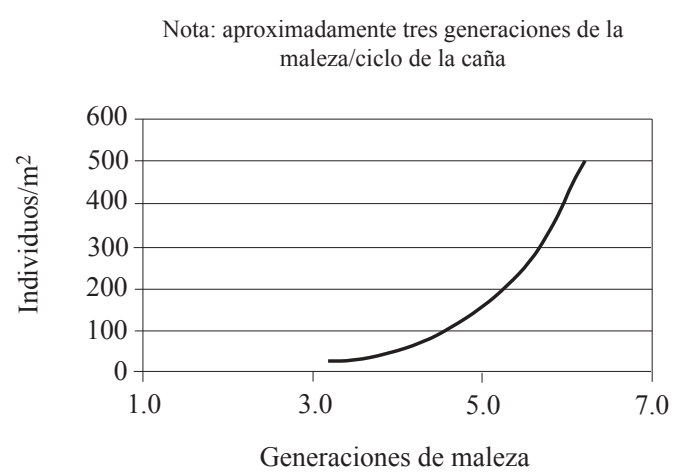

* Se asumieron 10000 semillas $/ \mathrm{m}^{2}$ (aprox. 750 semillas por planta adulta), de las cuales hasta un 5\% se pueden establecer como plántulas. Se asume también que de las 3 a 4 generaciones potenciales de la maleza por ciclo de la caña, sólo una escapa a los esfuerzos de control. Por último, a pesar de que se sabe que la viabilidad de la semilla varía entre 4 y 6 años, en esta versión preliminar no se toma en cuenta el efecto del banco de semillas.

Figura 2. Curva de reinfestación teórica de Rottboellia cochinchinensis bajo condiciones del Ingenio Taboga (modelo preliminar - versión simplificada/Arauz y Agüero, 2001).

cuentemente observados cuando los sistemas de labranza cambian. El sistema de labranza también influye en la distribución vertical y horizontal de semillas en el suelo, lo cual es importante ya que las semillas a diferentes profundidades en el perfil del suelo muestran diferencias en su regeneración (Ball 1992, Forcella 1992).

La labranza y la profundidad de las semillas en el suelo parecen constituir los factores más importantes en la densidad del banco de semillas y sobre las futuras poblaciones de malezas. Especies de malezas que tienen la habilidad para germinar y llegar a establecerse cuando las semillas están cerca de la superficie del suelo tienen un gran potencial para incrementar sus bancos bajo sistemas de labranza mínima.

\section{Clasificación de bancos de semillas}

Thompson y Grime (1979) dividen al banco de semillas en dos grupos:

El primer grupo consiste en bancos de semillas transitorios, que están constituidos generalmente por poaceas y no persisten en el suelo por más de un año, pues son producidas en una estación y germinan en el mismo año y generalmente permanecen cerca de la superficie del suelo. Por su corta existencia, son difíciles de examinar.

El segundo grupo consiste en el banco de semillas persistentes. Incluye especies que tienen un amplio ran- go de hábitats. Una porción de semillas permanece latente y llega a incorporarse, lo que constituye una estrategia de perpetuación. Cambia poco de estación a estación y por lo tanto se le puede examinar.

En las poáceas una mayoría del banco de semillas está localizado dentro de los dos primeros centímetros desde la superficie del suelo y toda la producción no suele abarcar más allá de los primeros $10 \mathrm{~cm}$ del suelo. En suelos cultivados la mayoría de las semillas se ubican en los primeros $15 \mathrm{~cm}$ del suelo, pero pueden encontrarse semillas a mayores profundidades mientras más profundo se cultive. Dorado et al. (1999) encontraron que el laboreo del suelo con arado deja un $25 \%$ de las semillas de malezas en los primeros $7 \mathrm{~cm}$ de profundidad y el resto hasta los $30 \mathrm{~cm}$. En cambio, la labranza reducida dejó $50 \%$ de las semillas en los primeros $7 \mathrm{~cm}$.

\section{Germinación y latencia}

La germinación es una reanudación del crecimiento del embrión que resulta en la emergencia de la plántula. La germinación puede ser epígea o hipógea. La primera consiste en la protusión de los cotiledones sobre la superficie del suelo y es más rápida que la germinación hipógea, en la cual los cotiledones permanecen bajo la superficie del suelo (Mayer y Poljakoff 1992).

Cuando finaliza la latencia, la secuencia lógica de eventos es el desarrollo de la radícula, lo cual indica que ocurrió la germinación, crecimiento bajo el suelo de las raíces primarias, salida de los brotes desde el suelo, y el establecimiento autotrófico de las plántulas, por el proceso de fotosíntesis. Una excepción a lo anterior ocurre cuando el arroz germina bajo condiciones de inundación; entonces, emerge primero el brote el cual se elonga con rapidez hasta la superficie para capturar y enviar oxígeno a través de los canales lisígenos a las raíces.

Para describir el establecimiento de las plántulas, Harper (1977) desarrolló el concepto del "sitio seguro", definido como un micrositio en el que la semilla encuentra condiciones apropiadas y seguras para la germinación. Desde este punto de vista, un análisis de laboratorio no es suficiente para predecir con exactitud lo que ocurre en el campo. La forma y tamaño de la semilla y el estado de la superficie del suelo parecen jugar un papel importante en determinar la disponibilidad de agua necesaria para una germinación exitosa (Silvertown 1982).

En realidad, los factores que influyen en la germinación son variados y complejos. Intensidad lumínica, fotoperiodo, luz, temperatura y sus fluctuaciones, nitratos, niveles de $\mathrm{O}_{2}, \mathrm{CO}_{2}, \mathrm{pH}$, humedad y abrasión física 
de la cubierta seminal son algunos de ellos (Silvertown 1982, Bello et al. 1995). La información acerca de los requerimientos del sitio seguro debe ser usada, para prevenir las infestaciones de las malezas y manipular el ambiente para promover o suprimir la germinación de malezas. Para el propósito de manejo de malezas es importante reconocerlas en estado de plántula. Las plántulas representan la etapa más vulnerable en el ciclo de vida de las malezas.

La latencia es el proceso por el cual las estructuras reproductivas de una especie (yemas, semillas, rizomas, tubérculos, etc) están viables, pero no germinan aunque sean puestas en condiciones óptimas de temperatura, humedad y oxígeno. La latencia es considerada el factor primordial que permite a las malezas perpetuarse en un suelo agrícola. Desde el punto de vista biológico es el estado de suspensión de la germinación que involucra diferentes factores fisiológicos y bioquímicos. La latencia y la germinación son procesos complejos interrelacionados por procesos bioquímicos y biofísicos y factores estructurales aún pobremente entendidos (Karssen 1982).

\section{Harper (1977) reconoce tres tipos de latencia:}

Latencia innata: ya existe cuando las semillas se desprenden de la planta madre. No germinarán aunque se den condiciones adecuadas para ello, hasta que ocurra un estímulo adecuado; por ejemplo, exposición a un periodo de estratificación en frío (Silvertown 1982). Esto garantiza a muchas especies evitar germinaciones suicidas durante la estación fría.

Latencia inducida: la latencia se puede inducir en semillas que nacieron sin ella, luego de que se entierren en el suelo o se expongan a la luz filtrada por follaje. La latencia se interrumpirá por exposición a la luz, cuando se disturba el suelo, o se abre un claro en la vegetación. En contraste con la latencia innata, que localiza la germinación en el tiempo, la latencia inducida restringe la germinación en espacio y tiempo (Silvertown 1982).

Latencia forzada: semillas que se encuentran bajo condiciones ambientales que impiden su germinación.

Sin latencia no habría problemas por malezas. Por mucho tiempo se ha aspirado a desarrollar productos que rompan la latencia de las semillas de estas plantas en el campo, de modo que se sincronicen germinaciones "suicidas". Herbicidas tiocarbamatos incrementaron la germinación de Abutilon sp y Chenopodium sp (Fawcett y Slife 1975). El gas etileno estimula la germinación de Striga asiatica y su uso permite reducir hasta un $90 \%$ el banco de semillas de esta especie pa- rasítica (Eplee 1975). Por ahora sin embargo, para la mayoría de las malezas parece más plausible el enfoque de evitar que se produzcan nuevas semillas, lo que Norris (2000) llama umbral de cero semillas (UCS).

Caver (1995) estudió la germinación en semillas enterradas de 12 especies. La mayoría de las semillas germinaron a una profundidad de $1 \mathrm{~cm}$ mientras que la germinación disminuyó a $20 \mathrm{~cm}$ de profundidad. La habilidad de las semillas de emerger a diferentes profundidades, depende del suelo y el peso de la semilla. Las semillas más grandes fueron capaces de germinar a mayores profundidades, por contener mayores reservas. Las propiedades físicas del suelo pueden retardar el desarrollo de las plántulas e influenciar la cantidad de luz que la semilla pueda recibir. Ha sido demostrado que la luz puede penetrar en un suelo franco arcillo limoso y franco arcillo arenoso hasta profundidades de $22 \mathrm{~mm}$. Semillas de Lactuca sativa expuestas a la luz equivalente a un día soleado, germinaron cuando estaban a 2 $\mathrm{mm}$ de la superficie del suelo, pero no así aquellas semillas enterradas a $6 \mathrm{~mm}$.

Por otro lado, es importante entender que, semillas que requieren un estímulo lumínico para germinar, lo pueden obtener en fracciones de segundo durante la labranza, aunque luego de esa práctica queden enterradas (Wilson 1988).

\section{Distribución horizontal de las semillas en el suelo}

Se han realizado numerosos estudios relacionados con la distribución vertical de semillas enterradas, pero hay escasa información acerca de la distribución horizontal de semillas en el suelo. No se conocen los mecanismos de dispersión horizontal de las especies que se encuentran en una determinada área de estudio ni las distancias que recorren sus estructuras de diseminación, por ello no es posible determinar si existe una distribución agregada de las semillas alrededor de la planta madre. El tipo de distribución debe conocerse para realizar una estimación confiable de la densidad de semillas de muestras tomadas al azar (Thompson 1986).

Las poblaciones pueden distribuirse de dos formas: 1) en forma agregada (contagiosa) con gran cantidad de individuos en algunas muestras y escasos o nulos en otras y 2) en forma uniforme (regular), que por lo general es difícil de encontrar en malezas.

La distribución agregada es muy común, con la característica que la varianza es mucho mas grande que la media (Roberts 1958, Goyeau y Fablet 1982). Greig (1983) encontró un excesivo número de muestras sin observar individuos presentes, mientras que en otras 
encontró una elevada densidad de individuos. Esto pudo deberse a: exclusión de especies de un área por factores ambientales desfavorables, presencia de especies competidoras, mecanismos ineficientes de dispersión de propágulos y propagación vegetativa. Benoit et al. (1989) determinaron un alto grado de agregación de semillas en el suelo, mientras que Johnson y Anderson (1986) encontraron que en sitios disturbados ocurren más parches.

Para una correcta elección del número de muestras suficientes para estimar la densidad de semillas en el suelo, lo deseable es conocer la distribución horizontal de las principales especies presentes en el banco.

\section{Distribución vertical de semillas en el suelo}

Las semillas en el suelo son usualmente más abundantes cerca de la superficie y su densidad declina rápidamente con la profundidad (Milton 1939, Harper 1977).

Leck y Graveline (1979) determinaron que el número de especies presentes disminuye con la profundidad, lo mismo mostraron los estudios de Major y Pyott (1966), y Strickler y Edgerton (1976). La declinación de la diversidad con la profundidad puede deberse a la compactación del suelo, ya que sólo las especies de semillas pequeñas pueden penetrar a mayor profundidad (Iverson y Wali 1982, Pareja et al. 1985).

Kellman (1970); Joung y Evans (1975) y, Stricker y Edgerton (1976) estudiaron por separado las muestras de broza (biomasa seca y hojarasca) y de suelo, encontrando un gran número de plántulas en la broza. Moree y Wein (1977) tomaron muestras, en los dos primeros centímetros del suelo incluyendo la broza presente en el mismo y encontraron la mayor densidad de plántulas a esta profundidad.

Thompson y Grime (1979) dividieron muestras de suelo en dos estratos: $0-3 \mathrm{~cm}$ (incluído la broza) y 3-7 $\mathrm{cm}$ de profundidad, y determinaron que las semillas enterradas a mayor profundidad integran en una alta proporción el banco de semillas "persistente". Johnson y Anderson (1986) encontraron un 66,5\% de emergencia de plántulas en el primer estrato $(0-2 \mathrm{~cm})$ y un $33,5 \%$ en el estrato inferior $(2-8 \mathrm{~cm})$. León y Agüero (2001) indican que el $90 \%$ de las semillas de Rottboellia cochinchinensis, una perniciosa poaceae de los trópicos, germina desde la superficie a los primeros $5 \mathrm{~cm}$ de profundidad, en cuatro suelos diferentes.

\section{Metodologías para determinar el tamaño del banco de propágulos y su potencial de regeneración}

La metodología para realizar el estudio de bancos de propágulos depende de los objetivos del mismo. A menudo se desean contrastar diferentes hábitats, prácticas de manejo, estudios en ecosistemas naturales disturbados y no disturbados, etc. A continuación se presenta una metodología para el estudio del banco de propágulos, potencial de regeneración y la lluvia de semillas. No se pretende ser extensivo, sino más bien proveer una herramienta metodológica simple, que incentive estos estudios en agroecosistemas.

\section{Determinación del tamaño del banco}

Para cuantificar el tamaño o densidad del banco de propágulos que se encuentran enterrados en el suelo, en general conviene realizar una demarcación de los sitios dentro del agroecosistema o ecosistema que se desee estudiar.

Es conveniente ubicar los puntos de muestreo en forma aleatoria en el lugar donde se ubicó el experimento.

Se sugiere tomar muestras de suelo hasta $20 \mathrm{~cm}$ de profundidad, pues ahí se encontrará la mayoría de los propágulos. Sin embargo, si se quiere determinar la distribución vertical, deben fijarse parámetros a diferentes profundidades en el perfil del suelo, lo cual dependerá de las malezas prevalecientes en el agroecosistema. Por ejemplo, en suelos con cultivos anuales la mayoría del banco de propágulos serán encontrados en la capa arable del suelo. En cambio, en agroecosistemas permanentes como cultivos perennes y pasturas dependerá del tipo de vegetación asociada con ellos.

Para la extracción de muestras del suelo se sugiere utilizar un barreno muestreador, entre más grande es la muestra más representativos serán los datos. Sin embargo, se debe reconocer que muestras muy grandes involucran un excesivo esfuerzo.

Las muestras obtenidas del banco deben ser transferidas a bolsas de polietileno debidamente marcadas y selladas para su inmediato traslado al laboratorio o al lugar donde se cuantificará el tamaño del banco.

Una vez que las muestras llegan al laboratorio se homogenizan y se dividen en sub - muestras iguales, para la realización de los diferentes ensayos, tales como potencial de regeneración, viabilidad, escarificación o estratificación, etc. 
Para cuantificar el tamaño del banco, un grupo de las submuestras se transfiere a bandejas (o macetas) de área conocida y cada muestra se pasa por una serie mínima de cuatro tamices o más $(0,15 ; 2,0 ; 4,0$ y 8,0 mm de diámetro) generalmente los diámetros exactos de los tamices se adecuan con base al tamaño de las estrategias de reproducción de las especies. Las sub-muestras se pasan por los tamices con la ayuda de agua a presión, hasta disolver completamente el suelo adherido a las estructuras de reproducción, teniendo cuidado de que no se pierda suelo fuera de los tamices por salpique.

El material retenido en cada tamiz debe ser identificado y analizado como las estructuras reproductivas (semillas, rizomas, tubérculos).

Los propágulos deben ser identificados y cuantificados para cada especie utilizándose lupas manuales y estereoscopios.

Se sugiere realizar el secado del material colectado de los tamices bajo condiciones naturales, aunque otros prefieren someter al secado en estufas, en este caso se corre el riesgo de dañar el embrion de la semilla cuando no se regula bien los gradientes de temperaturas.

Para la identificación y recuento de las estructuras reproductivas se utiliza una caja de Petri de base reticulada. La identificación taxonómica de las muestras recogidas se debe realizar con claves taxonómicas. Es necesario una identificación taxonómica precisa de las estructuras de diseminación de las especies con que se trabaja porque después se deberá cuantificarlas y reconocerlas mezcladas con el sedimento. Se recomienda trabajar primero con el reconocimiento de estructuras reproductivas originales, como aquellas que hayan completado su madurez fisiológica en la planta.

Debidamente identificadas, cada muestra se debe transferir a sobres de papel para su almacenaje en cámaras de refrigeración, cuando se desee realizar pruebas de tetrazolio, para determinar la viabilidad.

\section{Determinación del potencial de regeneración del banco}

Generalmente, cuando se quiere determinar el potencial de regeneración de una determinada especie, se refiere al componente activo del banco, o sea al aporte que tiene el banco en la brotación o germinación de sus disemínulos. En este caso, se sugiere homogenizar bien las muestras recolectadas y seccionarlas en dos partes iguales. Una parte de la muestra será para cuantificar el tamaño del banco y la otra para determinar el potencial de regeneración.
Las submuestras seleccionadas para determinar el potencial de regeneración deben ser transferidas a macetas o cilindros de área conocida.

El tamaño de la muestra debe ser igual a aquella obtenida para la cuantificación del tamaño del banco.

Las macetas con las muestras se deben colocar en forma aleatoria en un lugar determinado, en condiciones naturales de humedad, temperatura y luz, similares al sitio de muestreo.

Periódicamente, se debe proceder a la identificación taxonómica de las plántulas y brotes emergidos sobre la superficie del sedimento de las muestras, la identificación debe realizarse con la estructura de reproducción adherida a la plántula o brote.

Una vez hecha la identificación se debe proceder al conteo de plántulas y brotes emergidos, aquellos de identidad desconocida, se transferirán a otras bandejas hasta que la planta complete su ciclo, para luego realizar la identificación final.

La identificación se basa fundamentalmente en la forma, tamaño, color de hojas y tallos, flores y frutos que son característicos de cada especie.

Es recomendable establecer fechas fijas de evaluación y conteo de plántulas y brotes emergidos. Se sugiere continuar la evaluación hasta que no se observe emergencia de nuevos propágulos.

Si se quiere continuar con estudios de los propágulos no germinados es necesario tamizar las muestras de sedimento para obtener los propógulos que se encuentran latentes y luego con las pruebas de tetrazolio, determinar la viabilidad de los mismos.

\section{Determinación de la lluvia de semillas}

Una semilla de una maleza que ha llegado a su madurez fisiológica, constituye la vida potencial de una nueva planta. El destino de esa semilla depende de diversos factores endógenos y exógenos. Puede desprenderse ya latente de la planta madre y caer en la proximidad de ésta, desde dónde será transportada a distancias variables por factores bióticos o abióticos. Otras, en cambio, por su misma naturaleza, se producen dotadas de estructuras especializadas para la dispersión por diferentes agentes como el viento, agua, animales, etc. El papus en muchas asteráceas (ejm. Sonchus sp. y Cirsum sp.) es un ejemplo de lo anterior. Se ha publicado poco sobre la lluvia de semillas al suelo, quizás debido a lo complejo de predecir estas entradas. Un es- 
tudio realizado con Ruppia maritima en un sistema de irrigación en Argentina, mostró que el banco puede incrementarse hasta 22 veces en un sólo año (Acosta et al. 1999).

La metodología a utilizar para determinar la lluvia de semillas es complicada y se requiere un significativo esfuerzo. Si el estudio se realiza en invernadero en condiciones controladas resultará mas fácil, puesto que se puede controlar la producción de todas la estructuras reproductivas de una determinada especie. En condiciones de campo es más complicado debido a la influencia de los factores bióticos y abióticos, lo cual podrá afectar los resultados obtenidos. También, cuando se va a trabajar en condiciones de campo, la metodología puede diferir, dependiendo si se trata de cultivos anuales o perennes.

En el caso de cultivos perennes donde no existe remoción del suelo por un periodo de tiempo considerable, se sugiere fijar los puntos de muestro, colocándolos al azar durante la primera ocasión. Luego, con la ayuda de un barreno se debe extraer el suelo y en este sitio colocar un cilíndrico metálico inoxidable o de plástico con base perforada, para facilitar el escurrimiento del agua. Una vez colocado el cilindro en el suelo, su parte superior debe quedar al ras de la superficie del suelo. Es recomendable colocar una estaca en el centro del orificio del cilindro y asegurarlo bien en el sitio de muestreo, para evitar su movimiento por animales. Si se va a medir la influencia de distintos tratamientos en la lluvia de semillas es necesario ubicar estacas o puntos distintivos al lado del cilindro, con el objeto de facilitar su posterior evaluación. Luego, se fijarán las fechas de la recolección del sedimento que se depositará en los cilindros que fueron fijados, esto dependerá del tipo de estudio y de las condiciones climáticas de cada región, puesto que las malezas tendrán un comportamiento diferente con respecto a las condiciones climáticas. Para cuantificar los propágulos depositados en los cilindros se debe proceder al igual que para la determinación del banco de propágulos, anteriormente indicado.

Cuando se desee medir la lluvia de semillas por especie es necesario conocer bien la taxonomía de las semillas de cada maleza. Se sugiere realizar un levantamiento taxonómico de la vegetación establecida en el sitio de muestreo debido a que ésto ayuda a la identificación de los respectivos disemínulos.

\section{LITERATURA CITADA}

ACOSTA, L.W.; SABBATINI, M.R.; HERNANDEZ, L.F.; FERNANDEZ, O.A. 1998. Regeneración de cuerpos reproductivos de Potamogeton pectinatus, Ruppia maritima, Zannichellia palustris y Chara contraria: efecto de la temperatura. Phyton 63: 167-178.

ACOSTA, L.W.; SABBATINI, M.R.; FERNANDEZ, O.A.; BURGOS, M.A. 1999. Propagule bank and plant emergence of macrophytes in artificial channels of a temperate irrigation area in Argentina. Hydrobiologia 415: $1-5$.

ALTIERI, M. A.; LIEBMAN, M. 1988. Weed management in agroecosystems: ecological approaches. Boca Raton, Florida, CRC Press. 354 p.

ARCHIBOLD, O.W. 1980. Seed inputs as a factor in the regeneration of strip mine wastes in Saskatcheman. Can J. Bot. 58: 1490.

BALL, D.A. 1992. Weed seedbank response to tillage, herbicides, and crop rotation sequence. Weed Sci. 40: 645659.

BENOIT, D.L.; KENKEL, N.C.; CAVERS, P.B. 1989. Factors influencing the precision of soil seed bank estimates. Can. J. Bot. 67: 2833-3840.

BELLO, I.A.; OWEN, M.D.K.; HATTERMAN, H.M. 1995. Effect of shade on velvetleaf (Abutilon theophrasti) growth, seed production, and dormancy. Weed Tech. 9: 452-455.

BRUST, G.E.; HOUSE, G.J. 1988. Weed seed destruction by arthropods and rodents in low-input soybean agroecosystems. Am. J. Altern. Agric. 3: 19-25.

CAVER, P.B. 1995. Seed Bank: memory in soil. Can. J. Soil Sci. 75: 11-13.

COUSEN, R.; MORTINER, M. 1995. Processes involved in the regulation of population density. In: Dynamics of weed populations. Great Britain. Cambridge University Press. p. 86-134.

CRAWLEY, M.J. 1992. Seed predators and plant populations dynamics. In: Fenner, M. ed. Seeds: the ecology of regeneration in plant communities. Great Britain. CAB International. p. 157-191.

DYER, W.E. 1995. Exploiting weed seed dormancy and germination requirements through agronomic practices. Weed Sci. 43: 493-503.

DORADO, J.; DEL MONTE, J.P.; LOPEZ-FANDO, C. 1999. Weed seed bank response to crop rotation and tillage in semiarid agroecosytem. Weed Sci. 47: 67-73.

EPLEE, R.E. 1975. Ethylene: A witchweed seed germination stimulant. Weed Sci. 23: 433-436.

FAWCETT, R.S.; SLIFE, F.W. 1975. Germination stimulation properties of carbamate herbicides. Weed Sci. 23: 419. 
FENNER, M. 1995. Ecology of seed bank. In Galili, J.; Galili, G. eds. Seed development and germination. New York, Marcel-Dekker. p. 507-528.

FORCELLA, F. 1992. Prediction of weed seedling densities from buried seed reserves. Weed Res. 32: 29-38.

GREIG, P. 1983. Quantitative plant ecology: studies in ecology. Third Edition. Berkeley and Los Angeles. University of California Press. 359 p.

GOYEAU, H.; FABLET, G. 1982. Etude du stock de semences de mauvaises herbes dans le sol: le probleme del'echantillonnande. Agronomie 2: 545-552.

HARMON, G.W.; KEIM, F.D. 1988. The percentage and viability of weed seeds recovered in the feces of farm animals and their longevity in buried manure. In: Altieri, M.; Liebman, M. eds. Weed management in agroecosystems: ecological approaches. Boca Raton, Florida, CRC Press. 354 p.

HARPER, J.L. 1977. Population biology of plants. London, Academic Press. 892 p.

HARVEY, R. G.; BINNING, L.K. 1984. Importance of mourning dove in the spread of wild proso millet, Proc North Cent. Weed Control Conf. 39: 101.

IVERSON, L.R.; WALI, M.K. 1982. Buried viable seeds and their relation to revegetation after mining. J. Range Mang. 35: 648-653.

JOHNSON, R.G.; ANDERSON, R.C. 1986. The seed bank of a tallgrass prairi in Illinois. Am. Mild. Nat. 115: 123 130.

JOUNG, J.A.; EVANS, R.A. 1975. Germinability of seed reserves in a big sagebrush Community. Weed Sci. 235: 358-364.

KARSSEN, C.M. 1982. Seasonal patterns of dormancy in weed seeds. In: Khan, A., ed. The physiology and biochemistry of seed development, dormancy and germination. Elsevier, New York. p.243-270.

KELLMAN, M.C. 1970. The viable seeds content of some forest soil in coastal British Columbia. Can. J. Bot. 48: 1383-1385.

KELLY, A.D.; BRUNS,V.F. 1975. Dissemination of weed seeds by irrigation water. Weed Sci. 23: 486.

KIVILAAN, A.; BANDURSKI, R.S. 1981. The one hundredyear period for Dr. Beal seed viability experiment. Am. J. Bot. 68: 1290 .

KREMER, R. J. 1993. Management of weed seed banks with microorganisms. Ecol. Appl. 3: 24-52.
LECK, M.A.; GRAVELINE, K.J. 1979. The seed bank of a freshwater tidal marsh. Am. J. Bot. 66: 1006-1016.

LEÓN, R.; AGÜERO, R. 2001. Efecto de la profundidad del suelo en Rottboellia cochinchinensis (Lour) Clayton, en caña de azúcar (Saccharum officinarum L.). Agronomía Mesoamericana 12(1): 65-69.

MAJOR, J.; PYOTT, W.T. 1966. Buried, viable seeds in two California bunchgrass sites and their bearing on the definition of a flora. Vegetatio 13 (5): 253-282.

MAYER, A.M.; POLJAKOFF, A. 1982. The germination of seeds. 3rd ed. New York. Pergamon Press. 244 p.

MILTON, W.E.J. 1939. The occurrence of buried viable seeds in soils at different elevations and on a salty marsh. J. Ecol. 27 (1): 149-159.

MOREE, J.M.; WEIN, R.W. 1977. Viable seed populations by soil depth and potential site recolonization after disturbance. Can. J. Bot. 55: 2408-2413.

MURDOCH, A.J.; ELLIS, R.H. 1992. Longevity, viability and dormancy. In: Fenner, M. ed. Seeds: the ecology of regeneration in plant communities. Wallingford, Great Britain: CAB International. p. 193-229.

NORRIS, R.F. 2000. My view. Weed Science 48: 273.

PAREJA, M.R.; STANIFORTH, D.W., PAREJA, G.P. 1985. Distribution of weed seeds among soil structural units. Weed. Sci. 33: 182-189.

PARKER, V.T.; SIMPSON, R.L.; LECK, M.A. 1989. Patterns and processes in the dynamics of seed banks. In: Leck, M.; Parker, V.; Simpson, R. eds. Ecology of soil seed banks. San Diego, USA. Academic Press, p. 367384 .

POLLEN, S. 2000. Integrated weed management - Agronomy 320. Department of Agronomy. University of Wisconsin, USA. 31p.

ROBERTS, H.A. 1958. Studies on the weeds of vegetables crops: inicial effects of cropping on the weed seeds in the soil. J. Ecol. 46: 759-769.

ROBERT, G.H.; DOUGLAS, G.B. 1996. Weed seeds and the seed bank: implications for weed management. Extension and weed management, USDA-ARS, National Soil Tilth Lab Iowa State University. IPM 48: 1-6.

SILVERTOWN, J. 1982. Introduction to plant population ecology. London, Longman. 209 p.

STANELLE, J. 1988. Quality of 1984 Kansas wheat seed and its effect on quality and the yield of wheat, M.S. thesis, Kansas State University, Manhattan. In: Altieri, M.; Liebman, M. eds. Weed management in agroecosystems: ecological approaches. Boca Raton, Florida, CRC Press. 354p. 
STRICKLER, G.S.; EDGERTON, P. J. 1976. Emergent seedlings from coniferous litters and soil in eastern Oregon. Ecology 57: 801-807.

THOMPSON, K.; GRIME, J.P. 1979. Seasonal variation in the seed banks of herbaceous species in ten contrasting habitats. J. Ecol. 67: 893.

THOMPSON, K. 1986. Small-scale heterogeneity in the seed bank of an acidic grassland. J. Ecol. 74: 733-738.
YENISH, J.P.; DOLL, J.D.; BUHLER, D.D. 1992. Effects of tillage on vertical distribution and viability of weed seed in soil. Weed Sci. 40: 429-433.

WILSON, R.G. 1988. Weed seed biology in the soil. In: Altieri, M.; Liebman, M. eds. Weed management in agroecosystems: ecological approaches. Boca Raton, Florida, CRC Press. 354 p. 